\title{
A NEGLECTED POINT IN THE TEACHING OF ELEMENTARY DYNAMICS.
}

\author{
By Henry Crew.
}

Professor of Physics, Northwestern University, Evanston, Ill.

Few words in the English tongue have been made to carry so heavy a burden as the little monosyllable "force." No word is used more loosely or with a greater variety of meanings; yet no word is perhaps more capable of exact definition.

The following difficulty, which relates to this same physical quantity, is one which will appear to many as no difficulty whatever. But, having stumbled over it in my own teaching and having lcarned by conversation with other teachers that the question was by no means clear to all of them, I offer no further apology for mentioning it here.

Physical science, in so far as it has been reduced to a quantitative basis, is preéminent for its exact terminology. It defines physical quantities in a precise, clear-cut manner and then uses these definitions with rigid consistency.

From Newton to Poincaré inclusive the force acting upon any body at any instant has bcen defined as the product of the mass of the body multiplied by its acceleration at that instant. In terms of the usual algebraic symbols the defining equation of force is

$$
F=m a
$$

But if this be granted how is one to justify himself in saying that force is required to maintain a constant elastic deformation, where no acceleration of any kind is involved? This is the difficulty alluded to in the title of this note. If, for instance, one takes two ordinary spring balances, one in each hand, hooks them together and stretches them, each will register the amount of the pull. Since, however, no acceleration is present what right has one to infer the existence of a force? The patent reply is that "action and reaction are equal and opposite." Quite true: but if we are to accept mass-acceleration as the measure of force, what evidence have we that either of these forces-action or reaction-is present? Plainly we must either abandon mass-acceleration as the criterion of force or we must conclude that no force is acting in the case of the stretched spring. This suggests at once the solution of the difficulty, namely, mass-acceleration is the definition and measure. but not the criterion. of force. 
If, then, forces may exist where the accelerations are all zero, it is evident that the student must have a bridge connecting these two grotups of forces (viz: the so-called effective forces and other forces) so that he may employ the same unit, the dyne, whether working with one group or another. Some text books attempt to furnish such a bridge by attaching a mass to the lower end of a vertical spiral spring and using the weight of the suspended mass as a measure of the force required to elongate the spring. But here again there is no acceleration. The reply is,

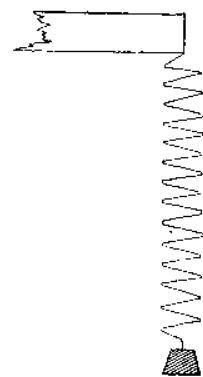
of course, that an acceleration would occur if the mass were allowed to fall freely. But when the mass is once detached the problem immediately becomes dynamical and hence very different from the statical solution offered.

The universal validity of our accepted definition of force should be illustrated by a direct and experimental connection between the force reFIG. 1 quired to maintain a constant elastic strain and the force required to maintain a constant acceleration in a given mass. Such an experiment is the following: Take an ordinary

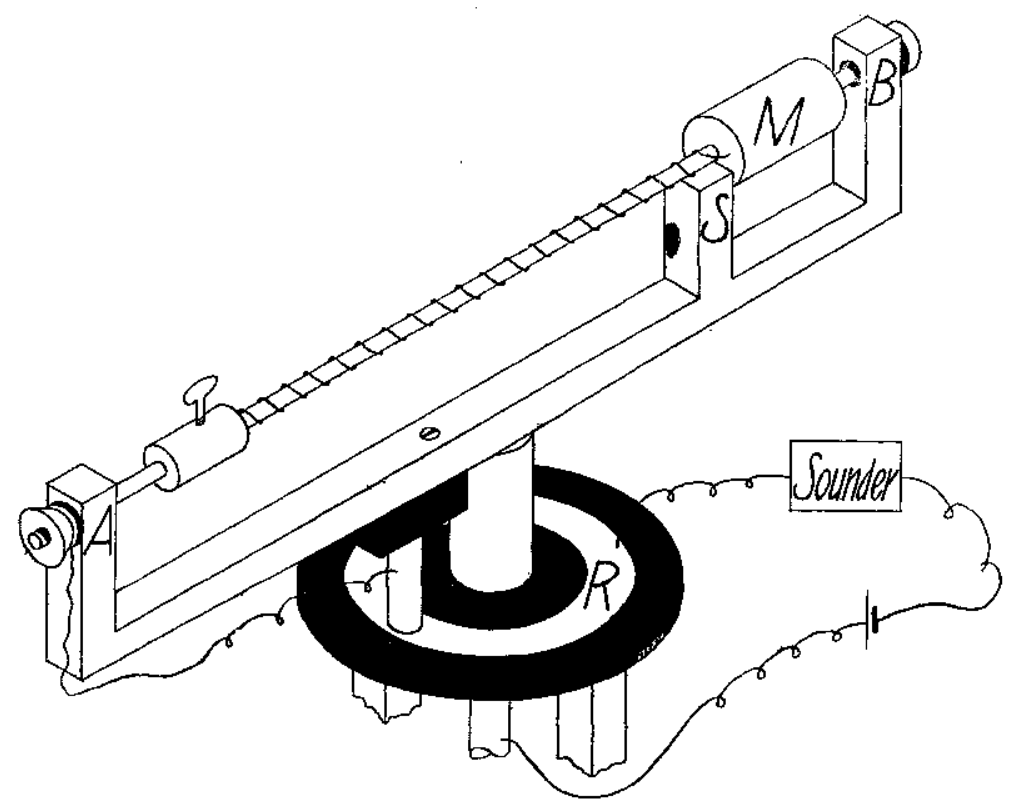

FIG. 2 


\section{Elementary Dynamics}

whirling table and slip over the steel rod, A B, a closely wound spiral spring of say No. 24 steel piano wire. Clamp onc end of the spring to the rod with a wire connector. To the other end attach a sliding mass of, say, 100 grams. When the table is rotated with an angular speed exceeding a certain amount the mass, M. will slide along the rod until it strikes the upright, B. The wooden stop, S, may be so placed as to prevent the mass, $M$, from slipping back more than, say, a single millimeter when the speed falls below the critical value necessary to keep the mass oscillating between the upright, $B$, and the stop, $S$.

It remains only to attach a bell circuit in such a way that one can easily detect the angular speed proper to keep the mass, $M$, teetering bctween the stops, $\mathrm{S}$ and $\mathrm{B}$. This is readily done as follows. Insulate the rod, A B, Fig. 2, by means of hard rubber bushings. Place a collector ring, insulated, of course, on the bearing in which the vertical shaft rotates. A brush which rotates with the table and bears on this ring may be used to connect the rod, $\mathrm{A} \mathrm{B}$, in series with a dry cell and a telegraph sounder, The other end of the electric circuit is joined to the vertical shaft of the whirling table, so that when the mass, M, touches $\mathrm{B}$ the circuit will be closed and the click of the sounder heard. To each position of the clamp there will be found a critical angular angular speed such that the circuit is being continually made and broken.

But to cach position of the clamp corresponds a definite elongation of the spring. Call the constant of the spring, $K$. Then to each critical speed $w$, corresponds an elongation $e$, such that

$$
K e=m r v^{2}
$$

where $r$ is the distance from the axis of rotation to the center of gravity of the mass, $m$.

Here we have an illustration of the connection desired; for here a force due to an elastic deformation is balanced against a massacceleration. The action, if we may so call the centrifugal aspect of the force, is dynamical: the reaction, that is, the centripetal aspect, is statical. In this manner the spring may in thought at least be calibrated throughout any desired range.

A more instructive experiment is perhaps the determination of the acceleration of gravity by this means. For this purpose 
it is only necessary to turn the table up on its edge and hold the rod, $A B$, at rest in a vertical position. Then set the clamp at such a point that the weight of the mass, $m$, is just sufficient to make it oscillate between the stops, $S$ and $B$. This position of the clamp is described by the following equation:

$$
K e=m g
$$

Now replace the table in its horizontal position and rotate it at such a speed-indicated again by the sounder-that we have

$$
K e=m r w^{2}
$$

Eliminating $K e / m$ between thesc two equations we have for our laboratory equation,

$$
g=r w^{2}
$$

Certainly no one will imagine this an exact method for the determination of " $g$ "; yet I have repeated the experiment sufficiently often to find that it is more accurate than the classical device of Atwood; at the same time it is more easily manipulated and is vastly more instructive to the student.

The upshot of the whole matter then is that, while mass-acceleration furnishes an excellent definition and measure of forcc, it is not a universally applicable criterion of force. Elastic deformations and other physical effects serve quite as well to indicate the presence of a force as does mass-acceleration.

May it not be well, therefore, while holding rigidly to the Nezetonian conception of force, to make it very clear to the student that the one physical feature which is probably characteristic of all forces is a state of stress; and that these various stresses gravitational, magnetic, electric, elastic, etc., niay be employed to equilitrate one another?

\section{LATITUDE AND THE LENGTH OF DAY.}

A LABORATORY EXERCISE.

Bx Josephe F. Morse, Medill High School, Chicago.

I. Almanac Study.

From an almanac answer the following questions for Chicago (New York or Boston) latitude:

(a) When are the days most nearly equal with the nights? (Equinox.) 